\title{
On-the-Fly Prediction of Orbit Corrections for RTK Positioning
}

\author{
Ahmed El-Mowafy \\ (The United Arab Emirates University) \\ (Email; Ahmed.Mowafy@uaeu.ac.ae)
}

\begin{abstract}
In this study, a method is presented to maintain real-time positioning at the decimetre-level accuracy during breaks in reception of the measurement corrections from multiple reference stations. The method is implemented at the rover by estimating prediction coefficients of the corrections during normal RTK positioning, and uses these coefficients to predict the corrections when reception of the corrections is temporarily lost. The paper focuses on one segment of this method, the on-the-fly prediction of orbital corrections. Frequently, only a few minutes of data representing short orbit 'arcs' are available to the user before losing radio transmission. Thus, it would be hard for the rover to predict the satellite positions using equations of motion. An alternative method is proposed. In this method, GPS orbital corrections are predicted as a time series and are added to the initial positions computed from the broadcast ephemeris to compute relatively accurate satellite positions. Different prediction approaches were investigated. Results show that the double exponential smoothing method and Winters' method can be successfully applied. The latter, however, has a better performance. The impact of the data length used for estimation of the prediction coefficients and the selection of seasonal lengths in Winters' method were investigated and some values were recommended. In general, the method can give orbital correction estimation accuracy of less than $5 \mathrm{~cm}$ after 15 minutes of prediction. This will result in a positioning accuracy better than $5 \mathrm{~cm}$.
\end{abstract}

\section{KEY WORDS}
1. Multiple Reference Stations.
2. Orbits.
3. Predictions.
4. Airborne Navigation.

1. INTRODUCTION. In real-time positioning, cm-level accuracy can be achieved using measurement corrections from a single or multiple-reference station network. However, this accuracy requires continuous reception of measurement corrections from the reference stations. In practice, one drawback of this approach is the occurrence of short breaks in real-time reception of the reference data. These breaks can range from a few seconds to some minutes. In standard ground surveying applications, the problem can be alleviated by waiting to resume work until reception has been regained. However, for reliable positioning in real-time kinematic applications, including precise airborne navigation, other solutions are needed to aid accurate positioning during reception gaps.

One method used to handle this problem is to integrate GPS with Inertial Measuring Units (IMU) in real time, as shown in El-Mowafy (2004). However, this 
solution is only valid for a short period due to the rapid deterioration of the IMU positioning accuracy in the standalone mode, as well as the increased cost and complexity of the hardware and software involved. Another solution would be to equip the receiver with wireless access to the Internet, so that precise orbit and clock products of short delays can be accessed from a service provider such as the Jet Propulsion Laboratory (JPL) or the International GNSS Service (IGS). In this case, the rover can operate in an un-differenced precise point positioning (PPP) mode utilizing both the code and carrier phase measurements and can achieve $\mathrm{cm}$ to decimetre accuracy, as shown by Gao et al. (2003) and Gao and Chen (2004). The shortfall of this technique is that it requires wireless connection, which is also subject to interruption.

2. THE PROPOSED METHOD. This study proposes another approach for GPS real-time positioning at the decimetre level without external aid (IMU, internet, etc.) in the event of temporary loss of the measurement corrections from multiple reference stations. In this method, un-differenced observables are used. At continuously operating multiple reference stations (RTK Reference Networks), all distance-dependent error components are modelled and separately estimated. Knowing the exact positions of the reference stations, the unknown vector at the reference stations $\left(\bar{X}_{r}\right)$ for $n$ number of observed satellites can be given as:

$$
\bar{X}_{r}=\left[c d T_{(t)},\left(d s, c d t, d_{\text {iono }}, \Delta_{W}, N, \varepsilon\right)_{1 . . n}\right]^{T}
$$

where $d s$ is the orbital error, $c$ denotes the speed of light, $d T$ and $d t$ are the receiver and satellite clock errors, $d_{\text {iono }}$ and $\Delta_{W}$ are the ionospheric and wet tropospheric delays respectively. $N$ is the integer phase ambiguity and $\varepsilon$ denotes the measurement noise including the multipath and the antenna phase centre variation. The zenith hydrostatic delay $\left(\Delta_{D}\right)$ component of the troposphere is estimated using the Saastamoinen model, and the satellite and station clocks are estimated using white noise stochastic models. A first order Gauss-Markov process is used for temporal correlations in the wet tropospheric errors. The use of multiple reference stations with continuous reception of satellite data, in addition to including data of the precise orbits from previous days and ultra-rapid orbits for the next day from the IGS website, facilitates having a fit for the satellites from the equations of motion at sufficient accuracy. The orbital errors are then computed at the reference stations as the difference between these satellite positions and the computed values from the broadcast message.

At the rover, during reception of the measurement corrections, a routine is run at relatively large time intervals to store the history and models, as a time series, the time change of each of the distance-dependent measurement corrections, for each satellite. These include corrections to the orbital error, the wet tropospheric error, and the satellite clock error. During this period, the prediction coefficients for each error are determined. When reception of the measurement corrections from the reference stations is interrupted, the corrections are independently predicted for several minutes ahead utilizing the estimated prediction coefficients. The rover receiver then works autonomously in a precise point-positioning mode using the un-differenced measurements after being corrected by adding the predicted corrections. An 
ionospheric-free measurement combination is used to eliminate the first order effect of the ionospheric error at the rover location.

The proposed method is based on the observation that errors usually change in time with some pattern, which the method tries to model in real-time and then use for error prediction. The discrepancies between the modelled patterns and the actual corrections represent the accuracy of prediction. Testing will show that these discrepancies can result in positioning accuracy at the decimetre level, which is one order of magnitude better than the only available approach of single point positioning in the absence of aiding tools (e.g. IMU, dead reckoning, and the internet). The main application considered for this technique is precise navigation and landing of aircraft (category III), as presented in El-Mowafy (2004), for which a vertical accuracy of $0.6 \mathrm{~m}$, and horizontal accuracy of $4.1 \mathrm{~m}$ are needed. Thus decimeter-level positioning accuracy, when achieved, would be sufficient. The significance of the proposed method is expected to increase after adding the third civilian frequency (Lc) due to an improvement in band protection, ionospheric error elimination, easy access to L2 signals, and increasing redundancy (Pervan et al. 2000).

The proposed method was evaluated using orbital corrections determined by the Dubai Virtual Reference System known as DVRS, in Dubai, UAE. The system consists of five permanent stations, constituting a multiple-reference station RTK network. Observations from the DVRS stations are gathered and processed in a common network adjustment at a central processing facility and measurement corrections are computed and sent to the rover. A user with a single receiver can use the network computed measurement corrections to estimate its position accurate to the centimetre-level, provided that a continuous reception of the corrections is maintained. If a break in this reception takes place, the accuracy will degrade, and will be similar to that of point positioning. This leads to the development of the above prediction process for measurement corrections. Details of the DVRS network and the achievable positioning accuracy using it can be found in El-Mowafy et al., 2003.

Since investigation of the time-series prediction of the three separately estimated and modelled distance-dependent errors including proof, tests, comparisons, results, and conclusions is too lengthy to fit in one paper, it is presented in three papers. This paper only investigates methods for on-the-fly prediction of satellite orbital corrections with the objective of achieving accuracy better than $10 \mathrm{~cm}$, which is acceptable for the application in hand. Investigations of the prediction methods for the other errors (clock errors and wet troposphere) are the subject of two other studies.

\section{PREDICTING THE ORBITAL CORRECTIONS IN REAL}

TIME. For the precise point positioning approach considered in this study, the orbital corrections are used, which are estimated with reference to the GPS orbits computed from the broadcast ephemeris. The orbital corrections are estimated in synchronization with any broadcast ephemeris updates, which typically take place every two hours. A suitable time interval (e.g. 5 seconds) can be used. The predicted orbital corrections at the rover are predicted from their previous values, which usually are computed for a short period of time (minutes to one hour). Therefore, long term patterns of 12 hours (for the $\mathrm{Z}$ satellite coordinate) and one day (for $\mathrm{X}$ and $\mathrm{Y}$ coordinates) that have been seen for instance in computations of the IGS precise orbits (Schenewerk 2003) cannot be detected at their full cycle. 
One should also note that the quality of the predicted corrections reflects the quality of estimation of the corrections at the rover before going to the prediction phase. This means that the presence of biased or erroneous corrections at some epochs during the period of estimating the parameters of prediction will result in wrong prediction modelling. This will cause incorrect predicted corrections for a long period. Therefore, data included in the time series has to be checked against such outliers. An empirical method for checking the presence of outliers can be formulated as:

$$
\left(d s_{t}-d s_{t-1}\right)<\zeta
$$

where $d s_{t}$ is the orbital corrections at time $t$, and $\xi$ is an allowable difference, which can be chosen as $3 \eta$, where $\eta$ is the mean value of the differences between consecutive corrections within a short window (e.g. 20 data points). If such an outlier is detected, it has to be removed from the prediction process. However, since time series analysis requires a continuous data sequence, the data values at missing epochs can be calculated by interpolation using polynomials or trigonometric functions, as applied in the standard interpolation process of the satellite orbits. This method has been discussed in several studies; see for instance Feng et al., 2004. In addition, in the case of receiving new broadcast ephemeris, large changes in the orbit corrections may be encountered. To check for the presence of major changes that may result in poor modelling, equation (5) can be applied. If the changes are significant, the prediction process has to be reset.

\section{CURRENT APPROACHES TO ORBIT ESTIMATION IN REAL-}

TIME APPLICATIONS. Estimation or prediction of satellite positions (orbit) can be generally performed based on the known force model (perturbations) acting on the satellite and the geometric constraints formed based on observing the satellite path. For real-time applications, the user usually employs the broadcast ephemeris, which results in predicted orbits of low accuracy. The standard error for these orbits is about $2.7 \mathrm{~m}$ (Springer and Hugentobler 2001), and thus a differential approach is usually needed to improve accuracy.

Alternatively, the user may use the more precisely predicted satellite positions from the IGS "ultra-rapid" orbits, which are derived from the GPS data recorded at numerous ground-based stations (Kouba and Héroux 2001). These predicted orbits can be obtained via the internet, and can give 3D estimates of satellite positions with a standard error in the 30 to $40 \mathrm{~cm}$ range (Soler and Snay 2004). The ultra-rapid orbits are provided at 15 minute intervals, four times a day, while the GPS broadcast ephemeris are updated every two hours. Thus, for a typical GPS processing, interpolation of the satellite orbits is needed at the required positioning interval.

One method of on-the-fly prediction of the satellite positions is to add the broadcast ephemeris to the orbital corrections to estimate precise orbits, and use this information to predict the satellite positions using the satellite equations of motion during the period of loss of the reference corrections. Computation of the satellite positions from the equations of motion can be found in several studies, see for instance Ash (2002). In the optimum conditions, satellite positions can be correctly predicted to the $\mathrm{cm}$ level. However, implementation of this approach for the case under investigation (a rover operating in a real-time airborne mode) is not a simple 


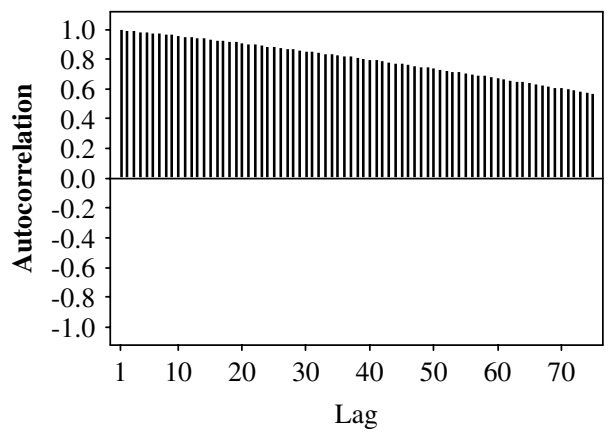

Figure 1. Autocorrelation function.

task. This is due to the fact that software using the equations of motion is relatively sophisticated, especially to make it run efficiently in on-board computers with limited processing power and memory. In addition, orbit fitting requires good estimation of parameters such as: satellite position, velocity, solar radiation pressure parameters, mass, surface area, general relativity effect, etc. Some of these parameters need to be regularly transmitted to the rover, which is currently hard to implement in practice as this requires changes in the length and format of the sent message. In addition, the orbit fitting process requires a considerable amount of orbital perturbation data for proper modelling and prediction. For the case in hand, it frequently happens that only a few minutes of data representing short orbit 'arcs' is available to the user. Fitting the parameters of the equations of motion correctly might then not be achievable under all circumstances for all satellites.

One simple approximation for prediction of the orbital corrections is to apply the coefficients derived from the standard interpolation methods such as Lagrange, Chebyshev, or Cowell's polynomials. However, all our tests showed that orbit prediction errors using these methods reached several decimetres after a few minutes of prediction. Thus, this approach is not suitable for prediction of satellite orbital corrections.

Thus, the main objective of this study is to develop an alternative simple and efficient approach that can achieve orbit prediction of accuracy less than $10 \mathrm{~cm}$, without the need for an internet connection or other aiding tools such as the IMU. This level of accuracy would be acceptable for the application in hand.

\section{PREDICTION OF THE ORBITAL CORRECTIONS BY TIME} SERIES ANALYSIS METHODS. In this method, the DVRS orbital corrections are predicted as a time series, based on the observation that satellite orbital estimation errors usually change in time with some pattern, which the method tries to model in real-time and use it for error prediction. The discrepancies between the modelled pattern and the actual corrections represent the accuracy of prediction. To select the best prediction methods, the autocorrelation and partial-autocorrelation functions of different satellite correction data sets were examined. To summarize findings, results of one typical example are given in Figures 1 and 2. As Figure 1 depicts, the autocorrelation functions for orbital corrections show large spikes at the beginning with subsequent positive autocorrelations that do not die down 


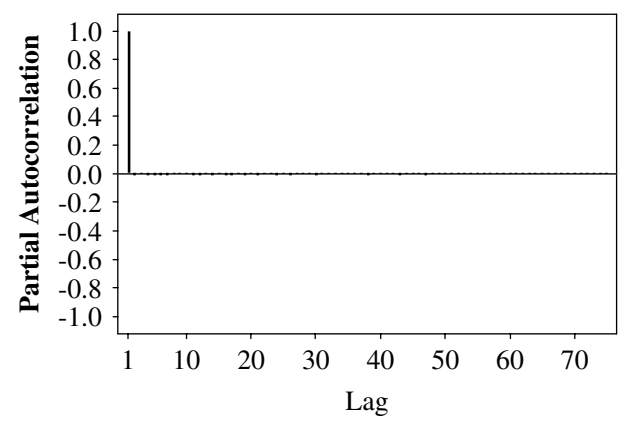

Figure 2. Partial autocorrelation function.

quickly. The partial autocorrelation function in Figure 2 shows a main spike at a lag of 1. From these patterns, as well as through visual inspection of different data sets, it can be concluded that three approaches can be applied successfully for prediction of orbital corrections as a time series. These are the quadratic autoregressive trend, the double exponential smoothing method, and Winters' method (HoltWinters' method).

The quadratic autoregressive trend model used for prediction of the orbital corrections at time $\mathrm{t}$ can be given as:

$$
d s_{t}=a+b t+c t^{2}
$$

where $a, b$ and $c$ are the model coefficients.

The double exponential smoothing method can be applied such that a level component (a constant) and a trend component are employed at each period. Two weights, or smoothing parameters, are used to update the two components (NIST/ SEMATECH 2005). The smoothing equations can then be written as follows:

$$
\begin{gathered}
L_{t}=\alpha d s_{t}+(1-\alpha)\left(L_{t-1}+T_{t-1}\right) \\
T_{t}=\gamma\left(L_{t}-L_{t-1}\right)+(1-\gamma) T_{t-1} \\
\widehat{d} s_{t}=L_{t-1}+T_{t-1}
\end{gathered}
$$

where $L_{t}$ and $T_{t}$ denote the level and the trend at time $t . \alpha$ and $\gamma$ are their weights, which are estimated by exponential smoothing. $\widehat{d} s_{t}$ is the fitted orbital correction, or one-step-ahead forecast, at time $t$. For the application in hand, equal weights can be assumed (e.g. 0·2).

As with the double exponential smoothing method, Winters' method employs a level component, a trend component at each period, and adds a third component representing seasonal changes (Chatfield and Yar 1991; Koehler et al. 1999). The method uses three weights to update the three components. Initial values for the level and trend components are obtained from a linear regression on time, whereas initial values for the seasonal component are obtained from a dummy-variable regression using de-trended data. The Winters' method smoothing equations in the 
multiplicative model adopted in our approach can be given as:

$$
\begin{gathered}
L_{t}=\alpha\left(d s_{t} / S_{t-p}\right)+(1-\alpha)\left(L_{t-1}+T_{t-1}\right) \\
T_{t}=\gamma\left(L_{t}-L_{t-1}\right)+(1-\gamma) T_{t-1} \\
S_{t}=\delta\left(d S_{t} / L_{t}\right)+(1-\delta) S_{t-p} \\
\widehat{d} s_{t}=\left(L_{t-1}+T_{t-1}\right) S_{t-p}
\end{gathered}
$$

where $L_{t}, T_{t}$ and $S_{t}$ are the level, the trend, and seasonal components at time $t . \alpha$, $\gamma$ and $\delta$ denote their weights. $p$ is the seasonal period. For simplicity, equal weights can be assumed, noting that large weights usually result in rapid changes in the model components.

6. INVESTIGATING PERFORMANCE OF THE TIME SERIES METHODS. The performance of the three prediction methods was investigated by comparing their differences in predicting orbital corrections with their correct values. The test data were computed from the multiple reference station DVRS Network. The outcome of studying a tenth of data sets can be summarized by presenting the results of four typical cases, which are illustrated in Figures $3 \mathrm{a}$ to $3 \mathrm{~d}$. Note the change of the vertical scale in Figures $3 \mathrm{c}$ and $3 \mathrm{~d}$. The statistics of the four cases are given in Table 1, which includes the average of the absolute values $(\mu)$ and the standard deviation $(\sigma)$ of the prediction errors estimated as the differences between the predicted and correct values. Results illustrated in Figure 3 are based on the use of 15 minutes of data to estimate the prediction coefficients and predict values of orbital corrections for the following 15 minutes using a data rate of five seconds.

Analysis of the first case illustrated in Figure 3a shows that errors of the quadratic autoregressive model grew rapidly with time, reaching $33.8 \mathrm{~cm}$ at the end of the prediction period. The errors of the double exponential smoothing model behaved similarly, but with lesser values. However, Winters' method gave the best results, as the errors in general were less than $10 \mathrm{~cm}$. Figure $3 \mathrm{~b}$ shows another case, in which the double exponential smoothing and Winters' methods had a similar behaviour, with errors of less than $2 \mathrm{~cm}$, while the quadratic autoregressive model errors grew to $20 \mathrm{~cm}$ at the end. Figure $3 \mathrm{c}$ illustrates a different case, in which the quadratic autoregressive model gave the best results. However, errors of the double exponential smoothing and Winters' methods were small in magnitude and did not exceed $4 \mathrm{~cm}$. The last case illustrated in Figure 3d shows that all three methods behaved almost similarly, with small errors that did not exceed $2 \mathrm{~cm}$. From these results, it can be seen that although the quadratic autoregressive model is the easiest method to implement, it gave poor results in several cases after a few minutes of prediction; hence, it cannot be generally used for prediction of the orbital corrections. One can also see that, except for the first case, the behaviour of the double exponential smoothing prediction method is close to that of the Winters' method, as they were almost equal, from the practical point of view, in the last three cases. However, the latter method for the majority of data sets gave the best prediction accuracy for the orbital corrections. Thus, it can be concluded that Winter's method is the best method that can be 
Table 1. Statistics of the four cases showing differences between the three investigated methods (m).

\begin{tabular}{|c|c|c|c|c|c|c|c|c|}
\hline & \multicolumn{2}{|c|}{ Case (a) } & \multicolumn{2}{|c|}{ Case (b) } & \multicolumn{2}{|c|}{ Case (c) } & \multicolumn{2}{|c|}{ Case (d) } \\
\hline & $\mu$ & $\sigma$ & $\mu$ & $\sigma$ & $\mu$ & $\sigma$ & $\boldsymbol{\mu}$ & $\sigma$ \\
\hline Quadratic & $0 \cdot 173$ & $0 \cdot 201$ & $0 \cdot 088$ & $0 \cdot 106$ & $0 \cdot 003$ & $0 \cdot 004$ & 0.0093 & $0 \cdot 0103$ \\
\hline Double Exp. & $0 \cdot 128$ & $0 \cdot 162$ & $0 \cdot 005$ & 0.007 & $0 \cdot 016$ & $0 \cdot 021$ & 0.0095 & $0 \cdot 0108$ \\
\hline Winters' & $0 \cdot 038$ & $0 \cdot 051$ & $0 \cdot 002$ & $0 \cdot 004$ & $0 \cdot 017$ & $0 \cdot 022$ & 0.0096 & 0.0107 \\
\hline
\end{tabular}

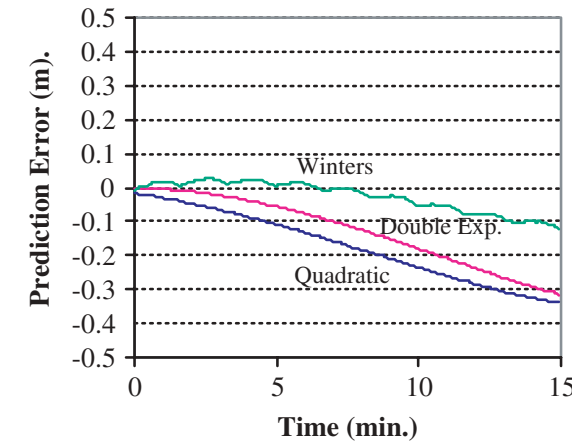

(a)

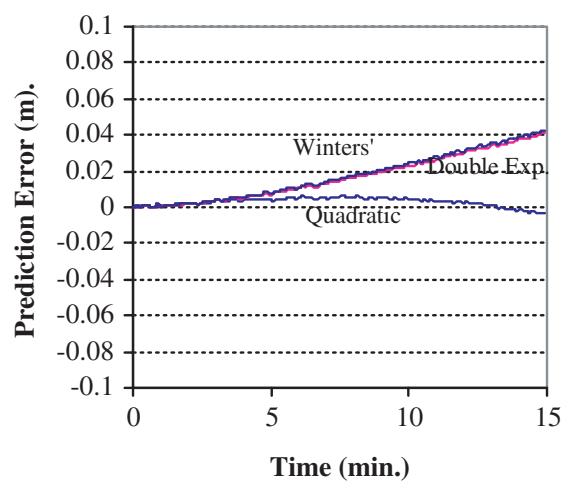

(c)

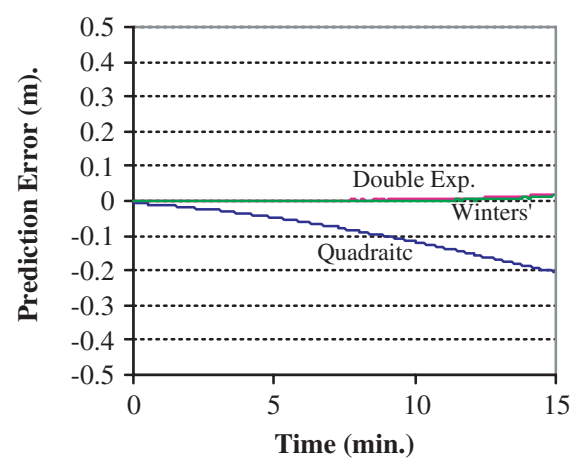

(b)

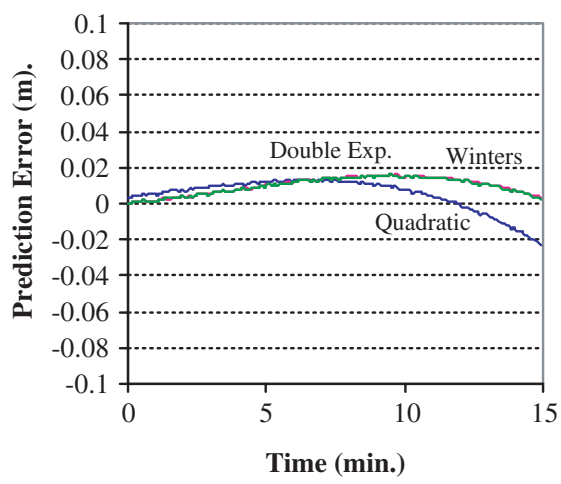

(d)

Figure 3. Typical cases showing accuracy of predicting orbital corrections using the three time series methods.

generally used for prediction of the orbital corrections. However, achieving accurate results with this method requires proper tuning of the seasonal length. This process is discussed in the next section.

\section{FACTORS AFFECTING PREDICTION IN WINTERS' METHOD.}

7.1. Length of the Data Used for Estimation of the Prediction Coefficients. The length of the data used for estimation of the prediction coefficients has a primary impact on the prediction accuracy and its pattern of change. This fact is illustrated in 

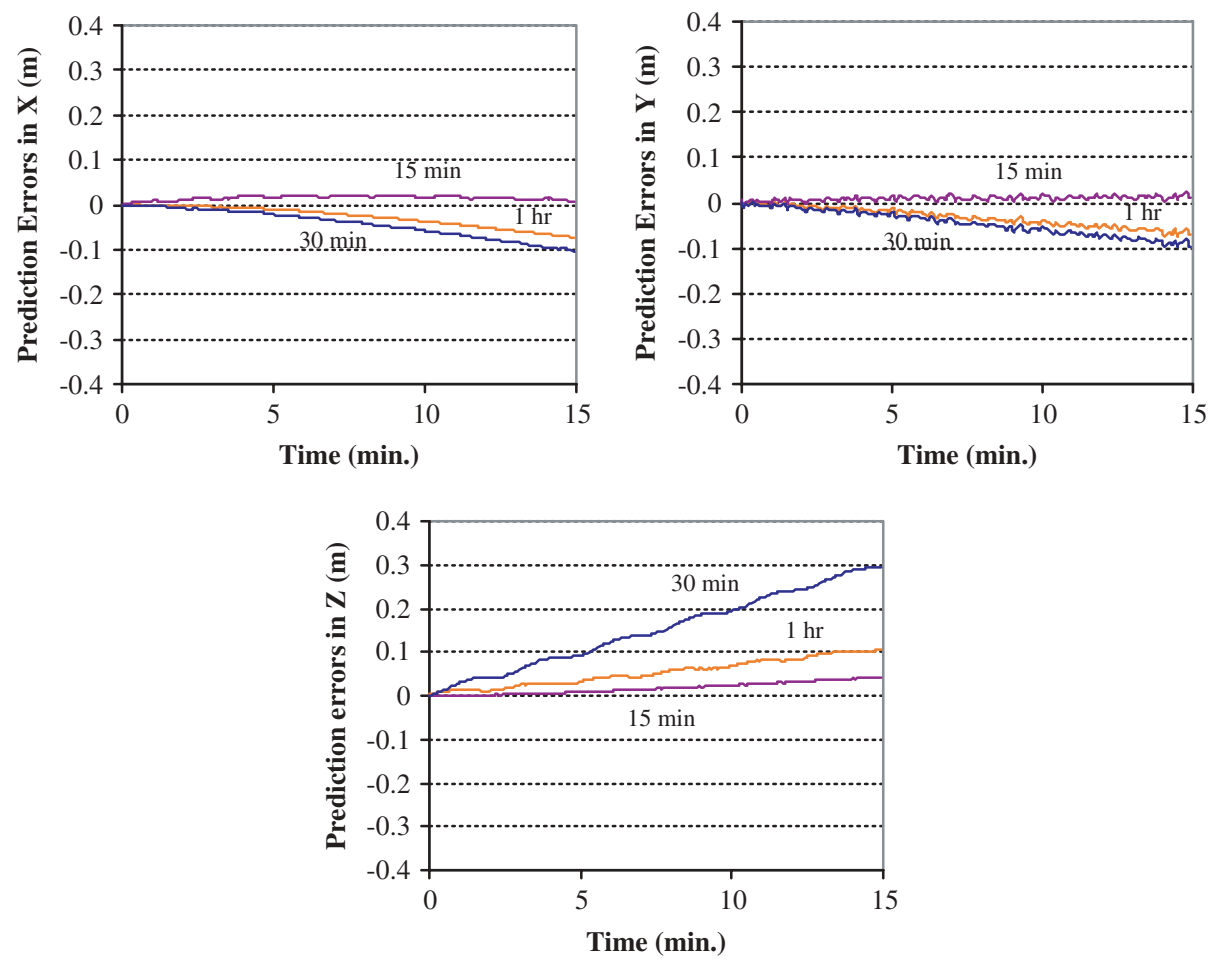

Figure 4. Prediction errors in $\mathrm{X}, \mathrm{Y}$ and $\mathrm{Z}$ coordinates using different data lengths.
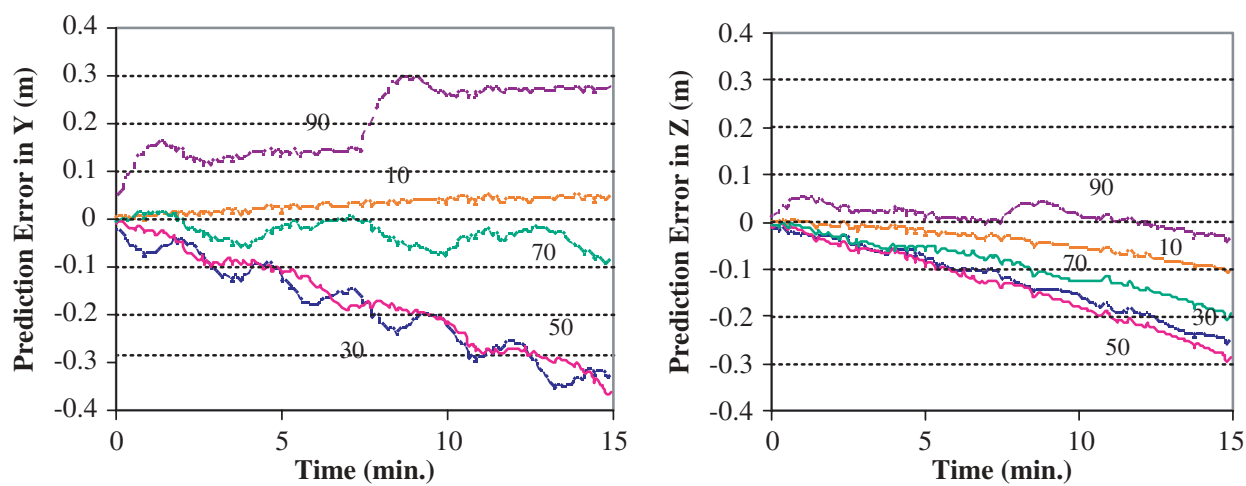

Figure 5. Prediction errors in $\mathrm{Y}$ and $\mathrm{Z}$ coordinates using different seasonal lengths in Winter's method.

Figure 4, which compares the use of 15, 30 and 60 minutes of data for estimating the prediction coefficients for the $\mathrm{X}, \mathrm{Y}$ and $\mathrm{Z}$ satellite orbital corrections. The figures show the prediction errors for a 15 minute period with a data rate of five seconds, for a typical case, in which data of satellite number 17, on January 26, 2005 is used. The prediction errors are computed as the differences between the predicted and previously known values of the orbital corrections, determined at the DVRS multiple reference station network. A seasonal length of 10 data points was employed when 
Table 2. Standard deviations of prediction orbital corrections using different data lengths (m).

\begin{tabular}{|c|c|c|c|c|c|c|c|c|c|}
\hline & \multicolumn{3}{|c|}{$\mathrm{X}$ coordinate } & \multicolumn{3}{|c|}{ Y coordinate } & \multicolumn{3}{|c|}{$\mathrm{Z}$ coordinate } \\
\hline & $15 \mathrm{~min}$ & $30 \mathrm{~min}$ & $1 \mathrm{hr}$ & $15 \mathrm{~min}$ & $30 \mathrm{~min}$ & $1 \mathrm{hr}$ & $15 \min$ & $30 \mathrm{~min}$ & $1 \mathrm{hr}$ \\
\hline SVN4 ${ }^{1}$ & $0 \cdot 013$ & $0 \cdot 007$ & $0 \cdot 012$ & $0 \cdot 030$ & $0 \cdot 032$ & $0 \cdot 018$ & $0 \cdot 014$ & $0 \cdot 007$ & $0 \cdot 013$ \\
\hline $\mathrm{SVN}^{2}$ & $0 \cdot 156$ & $0 \cdot 366$ & $0 \cdot 046$ & $0 \cdot 031$ & $0 \cdot 028$ & $0 \cdot 044$ & $0 \cdot 013$ & $0 \cdot 142$ & $0 \cdot 105$ \\
\hline SVN26 ${ }^{1}$ & $0 \cdot 010$ & $0 \cdot 119$ & $0 \cdot 072$ & $0 \cdot 006$ & $0 \cdot 051$ & $0 \cdot 001$ & $0 \cdot 022$ & $0 \cdot 174$ & $0 \cdot 061$ \\
\hline SVN26 ${ }^{2}$ & $0 \cdot 002$ & $0 \cdot 002$ & $0 \cdot 002$ & $0 \cdot 053$ & $0 \cdot 051$ & $0 \cdot 051$ & $0 \cdot 027$ & $0 \cdot 026$ & $0 \cdot 026$ \\
\hline SVN17 ${ }^{1}$ & $0 \cdot 004$ & $0 \cdot 048$ & $0 \cdot 004$ & $0 \cdot 011$ & $0 \cdot 053$ & $0 \cdot 038$ & $0 \cdot 019$ & $0 \cdot 038$ & $0 \cdot 020$ \\
\hline SVN17 ${ }^{2}$ & $0 \cdot 016$ & 0.053 & 0.035 & 0.028 & $0 \cdot 030$ & $0 \cdot 015$ & 0.067 & 0.067 & 0.069 \\
\hline
\end{tabular}

using a 15 minute estimation period, while in the cases of using 30 minutes and one hour, the seasonal lengths used varied between 20 to 50 data points. These values gave best results with the data lengths considered, as will be discussed in the next section. Table 2 gives statistical results for this comparison in terms of their error standard deviations. The table also gives similar comparisons for five additional tests to draw a better picture of the expected outcome. The standard deviations were computed directly from the prediction residuals for satellites numbers 4, 26 and 17, using two data sets for each satellite, which are separated by three hours.

Results from Table 2 show that a longer estimation period will not necessarily result in more accurate prediction of the satellite corrections. This is due to the nature of changes in this type of corrections. In general, the use of the recent 15 minutes for estimation of the prediction coefficients gave the best accuracy for most cases. Even when using one hour of data for estimating the model coefficients led to the best accuracy, the results of using 15 minutes were very close, mostly in the range of millimetres to a few centimetres. Similar results were seen in several tests carried out in this study.

7.2. Selection of the Seasonal Length. The seasonal length is dependent on the pattern of change of the data used. However, when using Winters' method in realtime applications, the choice of seasonal period should be automated. Different seasonal lengths can produce predictions with different accuracies. To illustrate this fact, Figure 5 shows prediction errors for the $\mathrm{Y}$ and $\mathrm{Z}$ coordinates of the satellite number 4, determined on February 17, 2005, using seasonal lengths of 10, 30, 50, 70 and 90 data points. For Y coordinates, seasonal lengths of 10 gave the best results, with errors less than $5 \mathrm{~cm}$, while for the $\mathrm{Z}$ coordinates a seasonal length of 90 gave the best results at the same error level. However, seasonal lengths of 10 still produce differences less than $10 \mathrm{~cm}$.

Optimal selection of the seasonal length for real-time processing can by done by storing the values of the orbital corrections for each satellite coordinate, and applying different seasonal lengths $(10,20,30, \ldots 90)$. The one that gives the least standard deviation can be chosen. The standard deviation can be computed from the differences between the fitted model values and the actual data. Alternatively, it can be computed for a short terminal period (e.g. 1-2 minute) from the differences between the predicted values using the tested seasonal length and their corresponding received 'correct' orbital corrections. This process can be performed while receiving the reference corrections, and thus it can be readily applied when the predicted values are needed. However, in practice, searching for the best possible seasonal length can be avoided when using short data lengths, as employing a seasonal length of 10 usually gives acceptable results for most cases. 
Based on studying different data sets, summarized in the last two sections, the recommended processing strategy for estimation of the prediction coefficients is to use all orbital corrections received for each satellite from start of reception of the reference corrections until loss of the data. If this period exceeds 15 minutes, data of the last 15 minutes only are used. A seasonal length of 10 data points can be used.

8. POSITIONING TESTING. Investigating the impact of predicting satellite orbital corrections on the accuracy of estimating the rover positions is of primary concern as it represents the main objective for developing this technique. For this purpose, several tests were carried out. However, to keep to a suitable manuscript length, only the results of two typical tests (in the sense that they represent the most common cases) are given. The test data were computed from the DVRS multiple reference station network. The tests were carried out using data from a rover receiver mounted on a moving vehicle. Positions of the rover receiver were determined using the precise point positioning approach employing both code and carrier phase data. Some details of this method can be found in Gao and Shen (2002). To evaluate positioning changes due to the prediction of satellite orbital corrections, the rover positions were first estimated using the correctly determined satellite orbit corrections, and then re-estimated in post-mission using the predicted orbit corrections. The results of the two sets of positions were then compared. The same remaining measurement corrections (e.g. satellite clocks and atmospheric corrections) were used for the two cases being compared, such that changes in positioning results will only reflect the use of the predicted orbital corrections. Six satellites were used in the first test, while eight satellites were used in the second. The test period considered was 15 minutes (6-6:15, Jan 26 for the first test, and 10-10:15, February 17, 2005 for the second test), with a positioning interval of five seconds. The data used as a source for estimating the predicted orbital corrections was the 15 minutes preceding the prediction period. A seasonal length of 10 was used in Winters' method for prediction of $\mathrm{X}, \mathrm{Y}$ and $\mathrm{Z}$ coordinates of all satellites, as it gave the least standard deviations of residuals between data of the fitted model and the correct data.

As an example, Figures 6 and 7 illustrate the first test positioning differences when using the correct and the predicted orbital corrections in the computed $\mathrm{X}, \mathrm{Y}$ and $\mathrm{Z}$ coordinates and the 3D positions of the test vehicle, respectively. Statistics of positioning discrepancies of the two tests are given in Table 3. The figures show that positioning errors were below $3 \mathrm{~cm}$ for the first five minutes. The errors grew with time, but they were generally below $5 \mathrm{~cm}$. Thus, the proposed method can be recommended for precise airborne navigation. Even in the case of receiving new broadcast ephemeris, with the possibility of encountering significant changes in the orbit corrections, the proposed method remains a better approach than to rely entirely on the single positioning mode.

9. CONCLUSIONS. A method is presented for prediction of the satellite orbital corrections on-the-fly as a time series during short breaks in reception of the multiple reference station data. The method is applied at the rover in the absence of external tools such as IMU or the internet. The proposed approach generally achieves positioning accuracy at the decimeter level. This accuracy is sufficient for 
Table 3. Statistics of positioning residuals due to prediction of orbital corrections (m).

\begin{tabular}{|c|c|c|c|c|c|c|c|c|}
\hline & \multicolumn{4}{|c|}{ Test 1} & \multicolumn{4}{|c|}{ Test 2} \\
\hline & $\mathrm{X}$ & $\mathrm{Y}$ & $\mathrm{Z}$ & $3 \mathrm{D}$ & $\mathrm{X}$ & $\mathrm{Y}$ & $\mathrm{Z}$ & $3 \mathrm{D}$ \\
\hline Average (Absolute) & $0 \cdot 012$ & $0 \cdot 017$ & $0 \cdot 021$ & $0 \cdot 033$ & $0 \cdot 014$ & $0 \cdot 013$ & $0 \cdot 020$ & $0 \cdot 029$ \\
\hline$\sigma$ & $0 \cdot 017$ & $0 \cdot 011$ & $0 \cdot 009$ & $0 \cdot 012$ & $0 \cdot 015$ & $0 \cdot 016$ & $0 \cdot 013$ & $0 \cdot 014$ \\
\hline Min. & $0 \cdot 001$ & $-0 \cdot 006$ & -0.001 & $0 \cdot 001$ & $0 \cdot 001$ & $-0 \cdot 002$ & $0 \cdot 002$ & $0 \cdot 001$ \\
\hline Max. & $-0 \cdot 050$ & $0 \cdot 044$ & $0 \cdot 039$ & $0 \cdot 061$ & $-0 \cdot 043$ & $0 \cdot 037$ & $0 \cdot 044$ & $0 \cdot 058$ \\
\hline
\end{tabular}

airborne navigation. The proposed method is simpler than the more accurate method of computing the satellite positions from its dynamics using equations of motion. However it avoids, in the required work environment, the possible difficulty the latter method may face in reaching an orbital fit from a limited period of data as well as the need to feed the rover with several data types related to the satellite's movement and the perturbing forces affecting its motion.

Different time series prediction methods were investigated. The double exponential smoothing method gave satisfactory results for most data sets and its results were close to the results from Winters' method. However, the latter technique is recommended for general use. Without the need for involved software that searches for the best estimation window and seasonal length, the user can satisfactorily use the data from the last 15 minutes (or less according to availability) to predict for the following 15 minutes using a seasonal length of 10 data points, assuming the use of a five second data rate. Orbital corrections with prediction errors of less than $5 \mathrm{~cm}$ can mostly be achieved. In general, positioning errors due to prediction of the orbital corrections were found to increase with time. However, they were usually below $5 \mathrm{~cm}$.

Modelling of the time series should avoid outliers in the data values as they can result in either a biased prediction or wrong modelling. Therefore, data included in the time series should be continuously checked. If outliers are detected, they should be removed and their values should be re-estimated using standard interpolation methods. Similarly, if significant changes in the orbit corrections are encountered after receiving a new broadcast ephemeris, estimation of the prediction coefficients has to be restarted.

\section{ACKNOWLEDGMENTS}

Dr. Nacho Romero, ESA, is sincerely acknowledged for his comments and valuable discussion. This study was financially supported by the Research Affairs Section, the UAE University. The Survey Section, Planning and Survey Department, Dubai Municipality is acknowledged for providing data from the Dubai Virtual Reference Network.

\section{REFERENCES}

Ash, M. E. (2002). Equipping GPS Satellites with Accelerometers and Satellite-to-Satellite Observables. Proceedings of the 2002 ION National Technical Meeting, San Diego, CA, 28-30 January.

Chatfield, C. and Yar, M. (1991). Prediction Intervals for Multiplicative Holt-Winters. International Journal of Forecasting, 7, 31-37.

El-Mowafy, A. (2004). Using Multiple Reference Station GPS Networks for Aircraft Precision Approach and Airport Surface Navigation. Proceedings of The 2004 International Symposium on GNSS/GPS, Sydney, Australia, 6-8 December. 


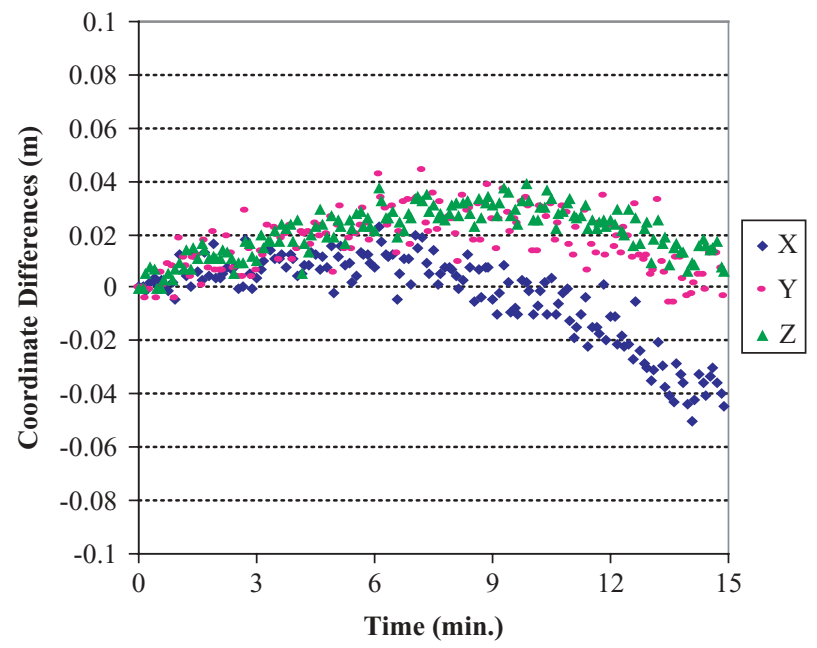

Figure 6. Errors in the rover coordinate due to prediction of the satellite orbital corrections.

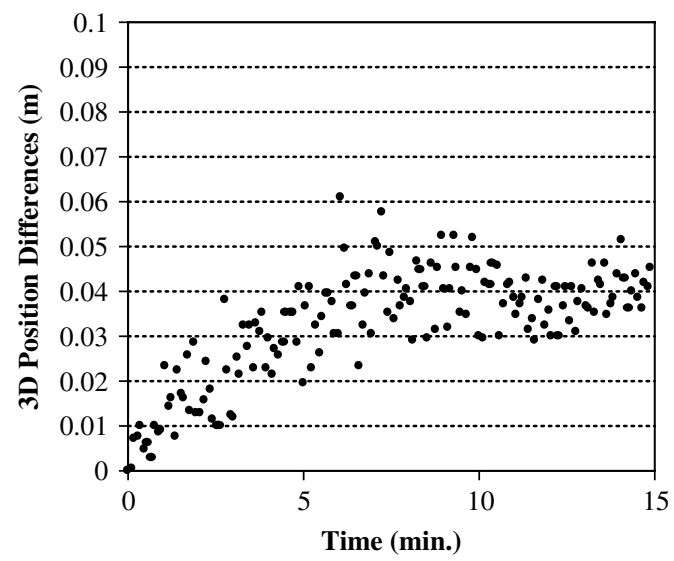

Figure 7. Errors in the 3D rover positions due to prediction of the satellite orbital corrections.

El-Mowafy, A., Fashir, H., Al Marzooqi, Y., Al Habbai, A. and Babiker T. (2003). Testing of the DVRS National GPS-RTK Network. Proceedings of the $8^{\text {th }}$ ISU International Symposium, Strasbourg, France, 25-28 May.

Feng, Y., Zheng Y. and Bai, Z. (2004). Representing GPS Orbits and Corrections Efficiently for Precise Wide Area Differential Positioning. Proceedings ION GNSS 2004, Long Beach, CA, 21-24 September.

Gao, Y. and Shen, X. (2002). A New Method for Carrier Phase Based Precise Point Positioning. Navigation, Journal of the Institute of Navigation, 49-2, 109-116.

Gao, Y., Chen, K. and Shen, X. (2003). Real-Time Kinematic Positioning Based on Un-Differenced Carrier Phase Data Processing. Proceedings of ION National Technical Meeting, Anaheim, California, 22-24 January.

Gao, Y. and Chen, K. (2004). Performance Analysis of Precise Point Positioning Using Real-Time Orbit and Clock Products. Proceedings of The 2004 International Symposium on GNSS/GPS, Sydney, Australia, 6-8 December. 
Koehler, A., Snyder, R. D. and Ord J. K. (1999). Forecasting Models and Prediction Intervals for the Multiplicative Holt-Winters Method. Working Paper 1/99, Department of Economics and Business Statistics, Monash University, Australia.

Kouba, J. and Héroux, P. (2001). GPS Precise Point Positioning Using IGS Orbit Products. GPS Solutions, 5-2, 12-28.

NIST/SEMATECH (2005). e-Handbook of Statistical Methods. http://www.itl.nist.gov/div898/handbook. Pervan, B., Jaewoo, J., Enge, P. and Hatch, R. (2000). Civilian GPS: The Benefits of Three Frequencies. GPS Solutions, 3-4, 1-9.

Schenewerk, M. (2003). A Brief Review of Basic GPS Orbit Interpolation Strategies. GPS Solutions, 6-4, 265-267.

Springer, T. A. and Hugentobler, U. (2001). IGS Ultra Rapid Products for (Near-) Real-time Applications. Phys. Chem. Earth, 26, 623-628.

Soler, T. and Snay, R. (2004). Transforming Positions and Velocities between the International Terrestrial Reference Frame of 2000 and North American Datum of 1983. Journal of Surveying Engineering (ASCE), 130-2, 49-55. 